\title{
Contribution of G protein-coupled estrogen receptor 1 (GPER) to $17 \beta$-estradiol-induced developmental toxicity in zebrafish
}

\author{
Graciel Diamante*, Norma Menjivar-Cervantes, Man Sin Leung, David C. Volz, \\ Daniel Schlenk*
}

Department of Environmental Sciences, University of California, Riverside, Riverside, CA 92507, United States

\section{A R T I C L E I N F O}

\section{Article history:}

Received 7 January 2017

Received in revised form 24 February 2017

Accepted 25 February 2017

Available online 2 March 2017

\section{Keywords:}

$17 \beta$-Estradiol

Zebrafish

Cardiac development

gper

Endocrine system and estrogenic

compounds

\begin{abstract}
A B S T R A C T
Exposure to $17 \beta$-estradiol(E2) influences the regulation of multiple signaling pathways, and E2-mediated disruption of signaling events during early development can lead to malformations such as cardiac defects. In this study, we investigated the potential role of the G-protein estrogen receptor 1 (GPER) in E2-induced developmental toxicity. Zebrafish embryos were exposed to E2 from $2 \mathrm{~h}$ post fertilization (hpf) to $76 \mathrm{hpf}$ with subsequent transcriptional measurements of heart and neural crest derivatives expressed 2 (hand2), leucine rich repeat containing 10 (Irrc10), and gper at 12, 28 and 76 hpf. Alteration in the expression of lrrc10, hand2 and gper was observed at $12 \mathrm{hpf}$ and $76 \mathrm{hpf}$, but not at 28 hpf. Expression of these genes was also altered after exposure to G1 (a GPER agonist) at 76 hpf. Expression of lrrc10, hand 2 and gper all coincided with the formation of cardiac edema at $76 \mathrm{hpf}$ as well as other developmental abnormalities. While co-exposure of G1 with G36 (a GPER antagonist) rescued G1-induced abnormalities and altered gene expression, co-exposure of E2 with G36, or ICI 182,780 (an estrogen receptor antagonist) did not rescue E2-induced cardiac deformities or gene expression. In addition, no effects on the concentrations of downstream ER and GPER signaling molecules (cAMP or calcium) were observed in embryo homogenates after E2 treatment. These data suggest that the impacts of E2 on embryonic development at this stage are complex and may involve multiple receptor and/or signaling pathways.
\end{abstract}

(c) 2017 Elsevier B.V. All rights reserved.

\section{Introduction}

Xenobiotics in the environment can alter the normal functions of the endocrine system by acting as antagonists or agonists to hormonal receptors, and/or alter the synthesis/metabolism of endogenous hormones (Guillette et al., 1995; Sonnenschein and Soto, 1998). For example, phenolic xenobiotic compounds structurally similar to the endogenous hormone, $17 \beta$-estradiol(E2), may alter the regulation of a variety of signal transduction events during development. Studies in zebrafish have been utilized extensively as a model to evaluate the effects of xenobiotics on development, and have shown that exposure to agents such as Bisphenol-A (BPA) and the natural hormone E2 during development resulted in a range of gross malformations, including curved body axis, yolksac edema, and pericardial edema (Brion et al., 2004; Duan et al., 2008; Saili et al., 2012). Similar cardiac phenotypes have been observed following polycyclic aromatic hydrocarbon (PAH) expo-

\footnotetext{
* Corresponding authors.

E-mail addresses: gdiam001@ucr.edu (G. Diamante),daniel.schlenk@ucr.edu (D. Schlenk)
}

sure in zebrafish embryos (Incardona et al., 2004). Although many studies have evaluated the impacts of xenobiotics such as PAHs on heart development, the molecular mechanisms by which estrogenic compounds cause cardiotoxicity is poorly understood.

Embryonic development depends on the precise spatiotemporal expression of signaling genes, and alterations can result in abnormal phenotypes. While nuclear E2 receptors have been primarily studied in development, limited work has been reported on the G-protein-coupled estrogen receptor 1 (GPER). Activation of GPER by G1 (a GPER agonist), E2 and other estrogenic compounds increases levels of secondary messengers such as $\mathrm{Ca}^{2+}$ (Brailoiu et al., 2013; Szego and Davis, 1967; Thomas et al., 2005), which can affect the expression of genes vital to cardiovascular development, such as Irrc10 and hand2 (Brody et al., 2013; Dirkx et al., 2013).

To better understand the effects of E2 and other potential phenolic contaminants that have estrogenic activities, the purpose of this study was to evaluate the role of GPER in the toxicity of E2 on zebrafish embryo development; G1 was used as a positive control for all exposures. To analyze the potential role of GPER in E2 and G1 toxicity, signal transduction and the expression of gper and cardiac genes, Irrc10 and hand2 were evaluated. 


\section{Materials and methods}

\subsection{Chemicals}

G1 (rel-1-[4-(6-Bromo-1,3-benzodioxol-5-yl)-3aR,4S,5,9bStetrahydro-3H-cyclopenta[c]quinolin-8-yl]-ethanone; $\geq 98 \%$ purity, Cayman Chemical, Ann Harbor, MI), G36 ((4S)-rel-4-(6-bromo-1,3-benzodioxol-5-yl)-3aR,4,5,9bStetrahydro-8-(1-methylethyl)-3H-cyclopenta[c]quinolone; $\geq 98 \%$ purity, Cayman Chemical); ICI 182,780182, 780 (7 $\alpha, 17 \beta$-[9-[(4,4,5,5,5-pentafluoropentyl)sulfinyl]nonyl]estra1,3,5(10)-triene-3,17-diol; $\geq 99 \%$ purity, Tocris, Minneapolis, MN) and $17 \beta$-estradiol (E2; $\geq 98 \%$ purity, Sigma-Aldrich, St. Louis, MO) were dissolved in ethanol. Stock solutions were kept at $4{ }^{\circ} \mathrm{C}$ in dark conditions. Exposure solutions were prepared by diluting working stocks in dechlorinated water at a final vehicle concentration of $0.1-0.2 \%$ ethanol within all vehicle control and treatment groups.

\subsection{Maintenance of zebrafish culture}

Zebrafish (Danio Rerio) 5D adults were purchased from Oregon State University. Adults were maintained and embryos were treated according to an animal use protocol (AUP \#20130005) that was reviewed and approved by the University of California, Riverside (UCR) Institutional Animal Care and Use Committee (IACUC). Adults were bred in tanks containing a mesh-spawning basket. The morning of treatment, embryos were collected 30 min after the light turned on. Embryos were cleaned and microscopically evaluated for viability. The stage of the embryos was visualized before treatment to ensure that exposure was conducted at a consistent developmental stage throughout all experiments. The different stages were determined using previously published standards (Kimmel et al., 1995). The embryos were maintained at $28^{\circ} \mathrm{C}$ in dechlorinated water with a light:dark cycle of $14 \mathrm{~h}: 10 \mathrm{~h}$.

\subsection{Exposure regime}

After microscopic evaluation, 30-35 randomly selected embryos were placed in petri dishes $(100 \mathrm{~mm} \times 15 \mathrm{~mm})$ for exposure. Treatment was initiated at $2 \mathrm{~h}$ post fertilization (hpf) and embryos were statically exposed until $76 \mathrm{hpf}$. At this time point, there is no cardiac progenitor cell formation which allows for sufficient time to determine the embryo viability without confounding cardiac effects. The embryos were exposed to $0.1 \%$ ethanol as the solvent control and to various nominal concentrations of E2 $(0.1,2$, $5,7$ and $8 \mu \mathrm{M})$. The concentrations were selected based on range finding studies and our own dose response experiment (data not shown). Each dish of treatment contained $20 \mathrm{ml}$ of the appropriate treatment solution. There were 4-9 replicates for each treatment depending on the specific experiment.

After exposure, the plates were incubated at $28^{\circ} \mathrm{C}$ in a light:dark cycle of $14 \mathrm{~h}: 10 \mathrm{~h}$. Embryos were statically exposed for $74 \mathrm{~h}$ and were checked daily for viability based on a transparency, yellowish appearance and presence of a heartbeat. Embryos that were determined to be dead were removed immediately at each observation time. At $76 \mathrm{hpf}$, treatment was terminated and surviving embryos were used for molecular endpoints (see below) and analyzed for abnormalities. Prevalence of the following gross malformations were assessed microscopically and quantified: curved body axis, bent tail, yolk-sac edema, looping defects and pericardial edema.

To investigate the role of GPER in E2-induced toxicity expression of GPER, Hand2, and Lrrc10 mRNA was analyzed at several time points during development including the segmentation (12 hpf) and pharyngula stages (28 hpf). We also analyzed expression of these genes and Vtg mRNA at the same time point morphological responses occurred (76 hpf). Vtg was included as a positive control for ER activation.

To determine and compare whether GPER activation resulted in similar effects as E2 during development, embryos were exposed to G1, a GPER agonist. Embryos were exposed at various concentrations $(0.5,2$ and $5 \mu \mathrm{M})$ of $\mathrm{G} 1$ at the same developmental time point as the E2 treatment. Doses were determined using range finding dose response experiments based on previous studies (Chandrasekar et al., 2010). To determine the contribution of GPER, co-exposure experiments were conducted with the GPER antagonist, G36 (5 $\mu \mathrm{M})$ and ICI 172, $780(14 \mu \mathrm{M})$. Concentrations of the antagonists were selected based on the solubility of these compounds in ethanol and the highest concentration that did not cause adverse effects on embryonic development, based on a range finding dose response experiment (data not shown).

\subsection{RNA isolation and RT-PCR}

Post-treatment embryos at 12,28 , and $76 \mathrm{hpf}$ were pooled for mRNA expression analysis. Approximately 35 embryos were pooled per treatment replicate $(n=4-9)$ and used for total RNA extraction using RNeasy Lipid Tissue Mini Kit purchased from Qiagen (Valencia, CA). After RNA extraction, the purity and integrity of the samples were analyzed using the $\mathrm{OD}_{260} / \mathrm{OD}_{280}$ ratio. Using the Promega Reverse Transcription System kit (Madison, WI), 1000 ng of RNA was used for cDNA synthesis following the manufacturer's instructions. Upon completion, cDNA was stored at $--20^{\circ} \mathrm{C}$ until qPCR was performed. qPCR was done using the iTAQ Universal SYBR green kit from BioRad (Hercules, CA). Each SYBR reaction mix had $100 \mathrm{ng}$ of cDNA and a specific primer set for the genes of interest. The primer sequence is as follows:

\section{gper forward-5' TGG CTG TGG CAG ATC TTA TTC 3' gper reverse-5' CAA TGG ACT GCT GCT CAT AGA 3' hand2 forward-5' AGA GAT GTC TCC TCC TGA CTA TAC 3' hand2 reverse-5' TTC CCT GAG TTC TGC AAA GG 3' Irrc10 forward-5' AGG AGC TTC CTC TGG TCA TA 3' lrrc10 reverse-5' AGC CTA AAT GGA GCG TCT TG 3' $v t g$ forward-5' CTG CAA GAG TGC AAC TGA TAG TTT C $3^{\prime}$ $v t g$ reverse-5' ACT TGC CAG TGA CTT TGT GCT T3' $e f-\alpha$ forward-5' CTA CAT CAA GAA GAT CGG CTA CAA $3^{\prime}$ ef- $\alpha$ reverse-5' CGA CAG GGA CAG TTC CAA TAC 3'}

The thermal cycling conditions used for all three genes analyzed via qPCR were as follows: The denaturation step was done at $95^{\circ} \mathrm{C}$ for $5 \mathrm{~min}$, followed by annealing and extension at $95^{\circ} \mathrm{C}$ for $10 \mathrm{~s}$ and at $55^{\circ} \mathrm{C}$ for $30 \mathrm{~s}$. This was repeated for 40 cycles. Afterward, a melt curve analysis was done from 54 to $95^{\circ} \mathrm{C}$ in increments of $0.5^{\circ} \mathrm{C}$. All primer sets displayed one peak demonstrating the specificity. Data was normalized using $e f-\alpha$ and the efficiency for all genes were optimized and monitored using PCRminer (Zhao and Fernald, 2005).

\subsection{Calcium measurements}

GPER signaling occurs through second messengers such as $\mathrm{Ca}^{2+}$ and cAMP (Brailoiu et al., 2013; Szego and Davis, 1967; Thomas et al., 2005). To measure intracellular $\mathrm{Ca}^{2}$, embryos after E2 and/or agonist/antagonist treatment were collected ( $\sim 30-35$ embryos per treatment replicate; $n=6$ ) and homogenized on ice using a lysis buffer $\left(150 \mathrm{mmol} \mathrm{L}^{-1} \mathrm{NaCl}, 1 \%\right.$ Triton $\mathrm{X}-100,0.5 \%$ sodium deoxycholate, $50 \mathrm{mmol} \mathrm{L}^{-1}$ Tris- $\mathrm{HCl}, 1 \mathrm{mmol} \mathrm{L}^{-1}$ EDTA, $1 \mathrm{mmol} \mathrm{L}^{-1}$ phenylmethanesulfonyl fluoride, $0.1 \%$ SDS). After incubation for $30 \mathrm{~min}$ on ice, lysed samples were centrifuged at $15,000 \times \mathrm{g}$ for $20 \mathrm{~min}$ at $4{ }^{\circ} \mathrm{C}$. The resulting supernatant was aspirated and used for the assay. Supernatant was mixed with $150 \mu$ l of the cal- 


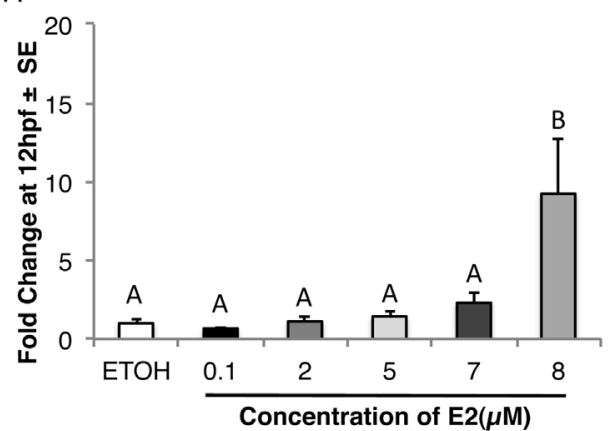

C

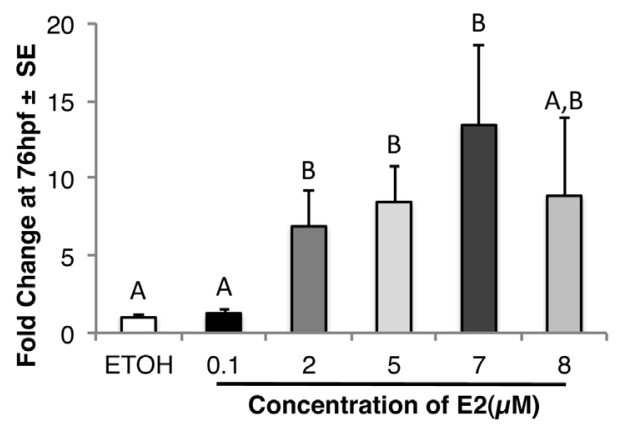

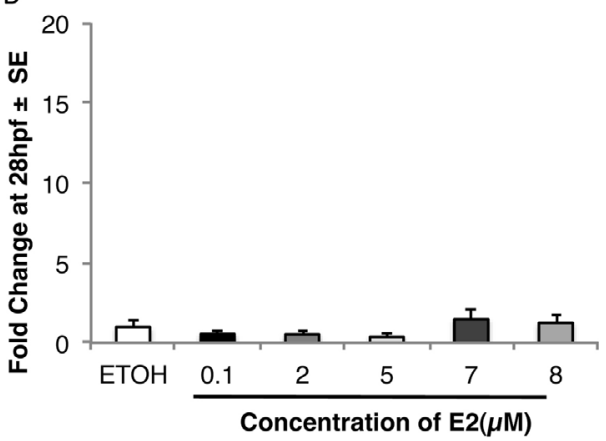

D

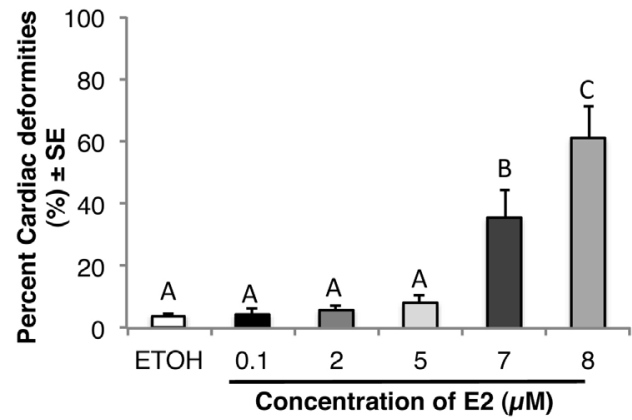

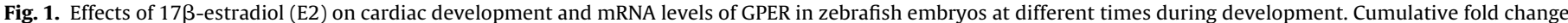

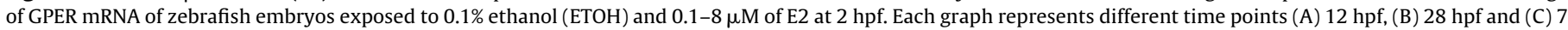

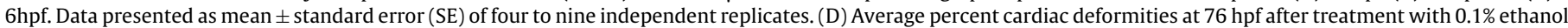

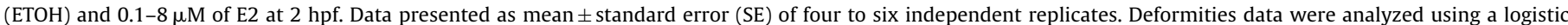

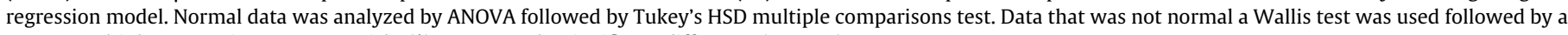
Dunn's Multiple Comparison Test. Asterisks $\left(^{*}\right)$ represent the significant difference $(p<0.05)$.

cium reagent Arsenazo III (Sigma Aldrich, St. Louis, MO) and the absorbance determined at $650-\mathrm{nm}$ with a spectrophotometer. The readings were then normalized to protein content, which was measured using a Pierce ${ }^{\mathrm{TM}}$ BCA Protein Assay Kit (Thermo Fisher Scientific, Waltham, MA) per manufacturer's instructions.

To measure cAMP, treated zebrafish embryos at $76 \mathrm{hpf}(30-35$ embryos per replicate, $n=4-6$ ) were snap-frozen in liquid nitrogen. cAMP concentrations was determined using Direct cAMP ELISA kit (Enzo Life Sciences, Farmingdale, NY) following the manufacturers' instructions. Proteins levels were determined using a Bradford assay (Thermo Fisher Scientific, Waltham, MA) for normalization.

\subsection{Statistical analysis}

All statistical analyses were done using the statistical program $R$ ( $R$ version 3.1.2). For parametric analyses, one-way ANOVA was used followed by a Tukey's HSD test $(p<0.05)$. Data sets that were not normally distributed were analyzed using a nonparametric method, the Kruskal-Wallis one-way analysis of variance. If the Kruskal-Wallis test showed significance $(p<0.05)$, then Dunn's post hoc test was used. Co-exposures were analyzed by a two-way ANOVA. Survival and deformity datasets were analyzed via logistic regression model.

\section{Results}

Sublethal malformations were observed after treatment with E2, including curved body axis, yolk-sac edema and pericardial edema (Supplemental Fig. 1). At $76 \mathrm{hpf}, 35.5 \pm 15.9 \%$ and $61.1 \pm 28.9 \%$ of the surviving embryos exhibited pericardial edema and cardiac impairment (such as looping defects) after exposure to $7 \mu \mathrm{M}$ and $8 \mu \mathrm{M}$ of E2, respectively, which were both statistically significant compared to all groups (Fig. 1A). To assess ER activation in embryos, $v$ tg expression was also analyzed at 76 hpf after treatment and an upward trend was observed following E2 treatment (Supplemental Fig. 2).

To investigate the role of GPER in E2-induced toxicity, expression of gper was analyzed at several time points during development including segmentation (12 hpf) and pharyngula stages (28 hpf). We also analyzed gper expression at the same time point morphological responses occurred (76 hpf). At $12 \mathrm{hpf}$, a 9-fold increase in gper expression was observed in the $8 \mu \mathrm{M}$ E2 treatment group (Fig. 1B). No significant changes were observed in gper mRNA levels at $28 \mathrm{hpf}$ (Fig. 1C). At $76 \mathrm{hpf}$, when abnormalities were observed, a significant increase in gper expression occurred after exposure to $2 \mu \mathrm{M}$ (6.9-fold increase), $5 \mu \mathrm{M}$ (8.5-fold increase) and $7 \mu \mathrm{M}$ of E2 (13.4-fold increase), respectively (Fig. 1A and D).

The expression of two cardiac genes lrrc10 and hand2 regulated by GPER through $\mathrm{Ca}^{+}$and cAMP signaling also increased after exposure to E2. The patterns of $\operatorname{lrc} 10$ and hand2 expression were correlated with an $r^{2}$ value of 0.88 . Expression of lrrc10 transcripts at $12 \mathrm{hpf}$ showed an increasing trend after exposure to $5 \mu \mathrm{M}$ and $7 \mu \mathrm{M}$ of E2, with a significant 9.6-fold increase observed at $8 \mu \mathrm{M}$ E2 (Fig. 2A). At $28 \mathrm{hpf}$, Irrc10 expression did not significantly change (Fig. 2B). At $76 \mathrm{hpf}$, an upward trend in $\operatorname{lrc} 10$ expression was seen after exposure to 2, 7 and $8 \mu \mathrm{M}$ of E2, with a significant change at $5 \mu \mathrm{M}$ exposure (Fig. 2C). At $12 \mathrm{hpf}$, the expression of hand 2 after exposure to $8 \mu \mathrm{M}$ of E2 resulted in a significant 5.7-fold increase (Fig. 3A). At $28 \mathrm{hpf}$, hand2 expression did not significantly change (Fig. 3B). At $76 \mathrm{hpf}$, hand2 expression showed an upward trend after exposure to 2,7 and $8 \mu \mathrm{M}$ of $\mathrm{E} 2$, but a significant change only occurred at $5 \mu \mathrm{M}$ exposure (Fig. 3C).

After exposure to $2 \mu \mathrm{M}$ and $5 \mu \mathrm{M}$ of $\mathrm{G} 1$ for $74 \mathrm{~h}$, there was a significant increase in cardiac deformities (e.g., pericardial edema and looping defects). Treatment with $2 \mu \mathrm{M}$ and $5 \mu \mathrm{M}$ of $\mathrm{G} 1$ resulted in $87.7 \pm 19.4 \%$ and $100 \pm 0 \%$ of embryos with cardiac deformi- 
$12 \mathrm{hpf}$

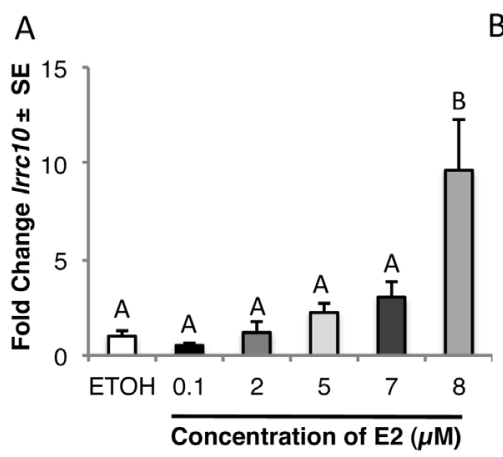

$28 \mathrm{hpf}$

B

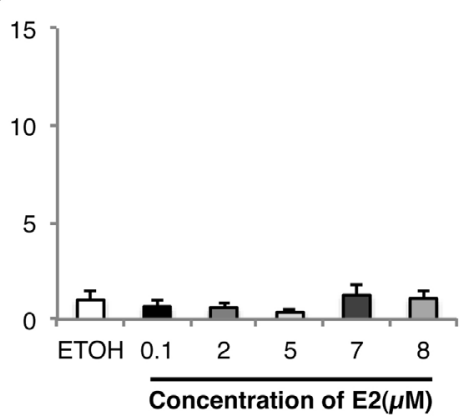

$76 \mathrm{hpf}$

C

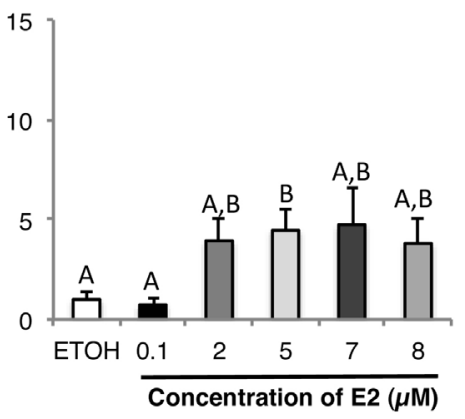

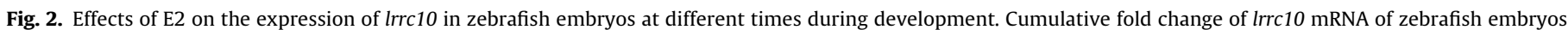

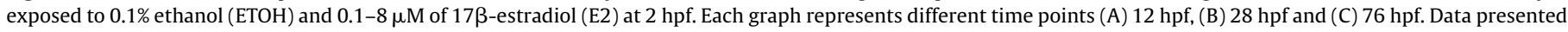

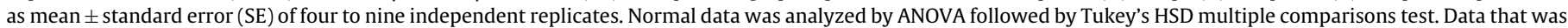

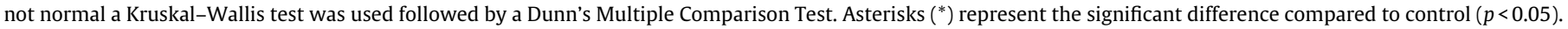

$\begin{array}{llll} & & & \\ \text { B } & \text { 28hpf } & \text { C } & \end{array}$

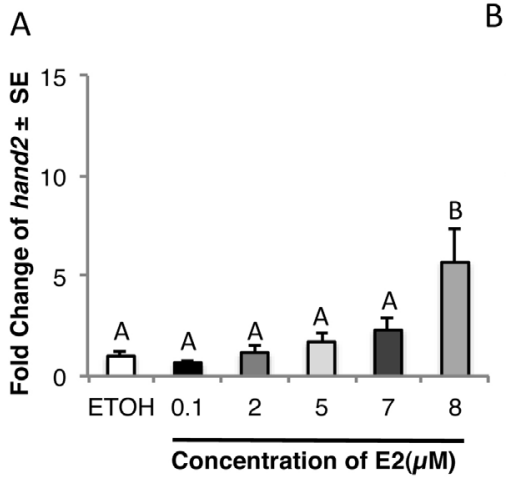

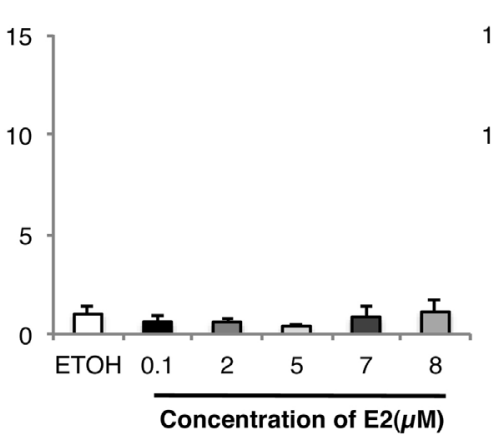

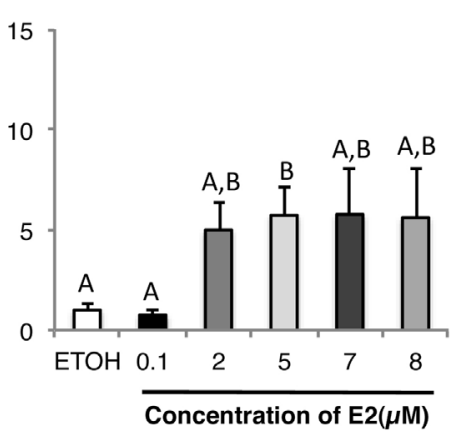

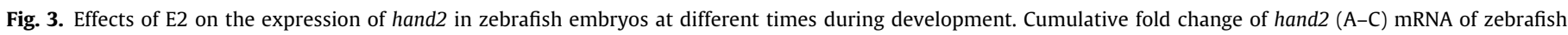

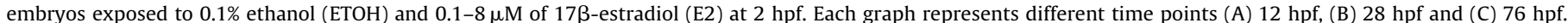

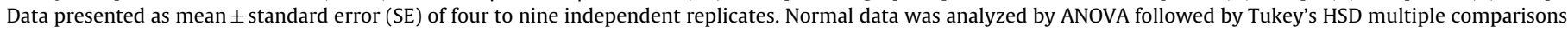

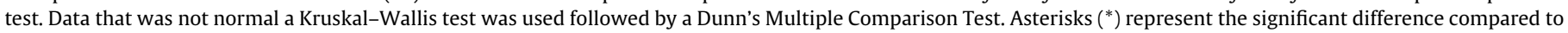
control $(p<0.05)$.

ties (Fig. 4A). After treatment with $2 \mu \mathrm{M}$ of $\mathrm{G} 1$, expression of Irrc10, hand2 and gper were significantly induced. Expression of gper resulted in a 9-fold increase compared to the vehicle control (Fig. 4B). G1 exposure also resulted in a 8- and 9-fold increase in the expression of $\operatorname{lrc} 10$ and hand2, respectively (Fig. 4C and D).

To assess whether the impacts on cardiac development were mediated through activation of GPER, E2- and G1-exposed embryos were co-exposed with the GPER antagonist G36. Exposure up to $5 \mu \mathrm{M}$ of $\mathrm{G} 36$ did not cause cardiac deformities. Embryos exposed to $7 \mu \mathrm{M}$ of E2 and $1.75 \mu \mathrm{M}$ of G1 both caused a significant increase in the percentage of embryos that had cardiac deformities $(45.4 \pm 5.3 \%$ and $52.8 \pm 6.1 \%$ respectively). Co-exposure of embryos to $\mathrm{G} 1$ and G36 decreased the abnormalities observed with $\mathrm{G} 1$ to $13.3 \pm 2.5 \%$ (Fig. 5A). In addition, the alterations observed in gper, lrrc10 and hand 2 transcripts after $\mathrm{G} 1$ exposure were also abolished (Fig. 5B and C). This suggests that expression of these genes may be associated with GPER-induced cardiotoxicity.

The increase in the expression of gper, Irrc10 and hand2 observed in the E2 exposed embryos were reduced when co-exposed with G36 (Fig. 6B and C). However, the deformities were not rescued in the embryos that were co-exposed with E2 and G36 (Fig. 6A). Since E2 deformities were not rescued by G36, we investigated whether the nuclear estrogen receptors were involved in E2 developmental toxicity. Embryos were co-exposed to $14 \mu \mathrm{M}$ of ICI, 182,780 (ICI). The co-exposed samples did not significantly decrease the num- ber of observed deformities, although levels of $v t g$ expression was diminished confirming the concentration of ICI necessary for ER antagonism (Fig. 7 and Supplemental Fig. 2).

Activation of GPER enhances levels of intracellular secondary messengers such as cAMP and $\mathrm{Ca}^{2+}$, which can moderate the expression of Irrc10 and hand2 (Brody et al., 2013; Dirkx et al., 2013). Levels of both secondary messengers were analyzed after exposure to 2, 5 and $7 \mu \mathrm{M}$ of E2, and the GPER agonist G1. There was an increase in the levels of $\mathrm{Ca}^{2+}$ following treatment with $5 \mu \mathrm{M}$ of G1. However, significant changes were not seen after exposure to E2 for $74 \mathrm{~h}$ at any of the concentrations analyzed (Supplemental Fig. 3). There was also no increase in cAMP after treatment with E2 and a trend towards a decrease after G1 exposure (Supplemental Fig. 4).

\section{Discussion}

Activation of GPER by the GPER agonist G1 induces similar malformations as those of embryos exposed to other estrogenic compounds (Jayasinghe and Volz, 2012). Binding of E2 or G1 to GPER can alter transcriptional regulation of genes required for development. In the present study, the role of GPER on cardiac impacts by E2 during development was evaluated. We showed that exposing zebrafish embryos to E2 and G1 during embryogenesis altered the transcription of gper and genes involved in cardiac 
A

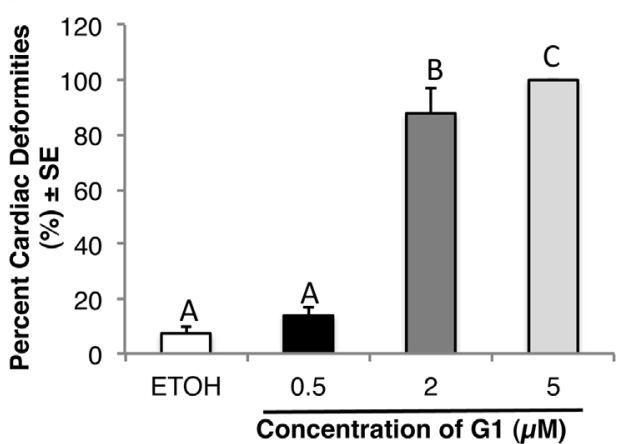

C

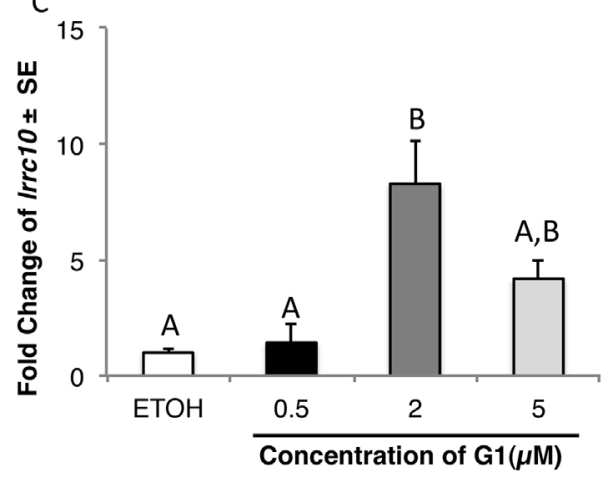

B

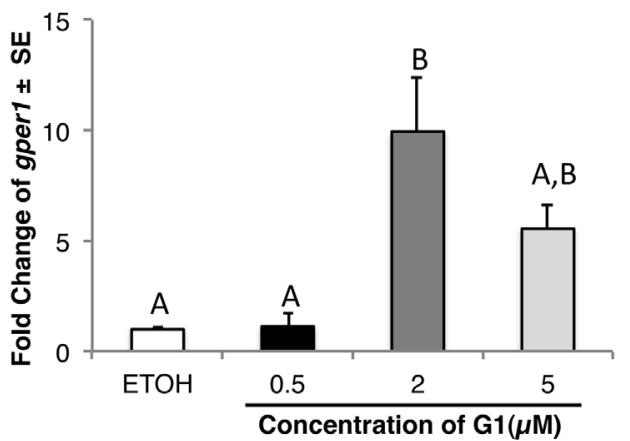

D

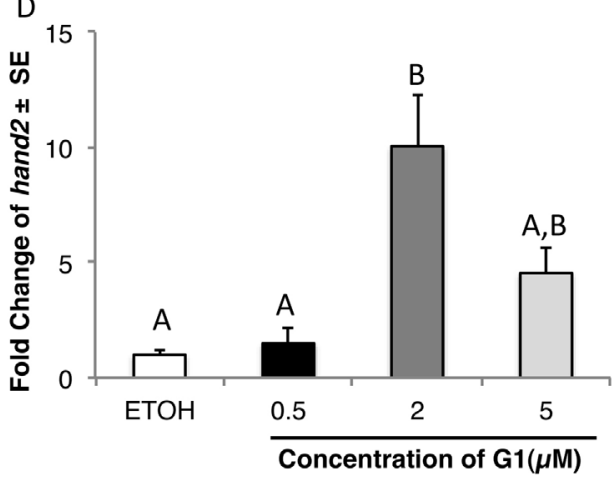

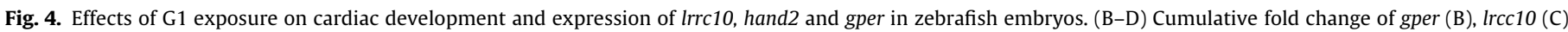

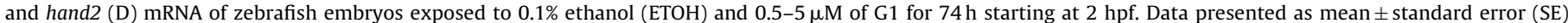

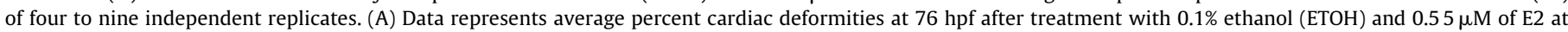

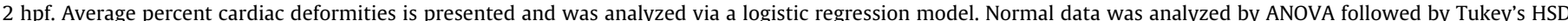

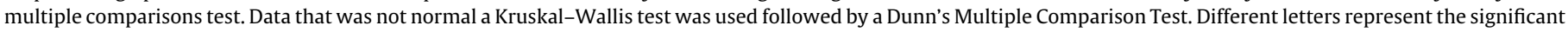
differences $(p<0.05)$.
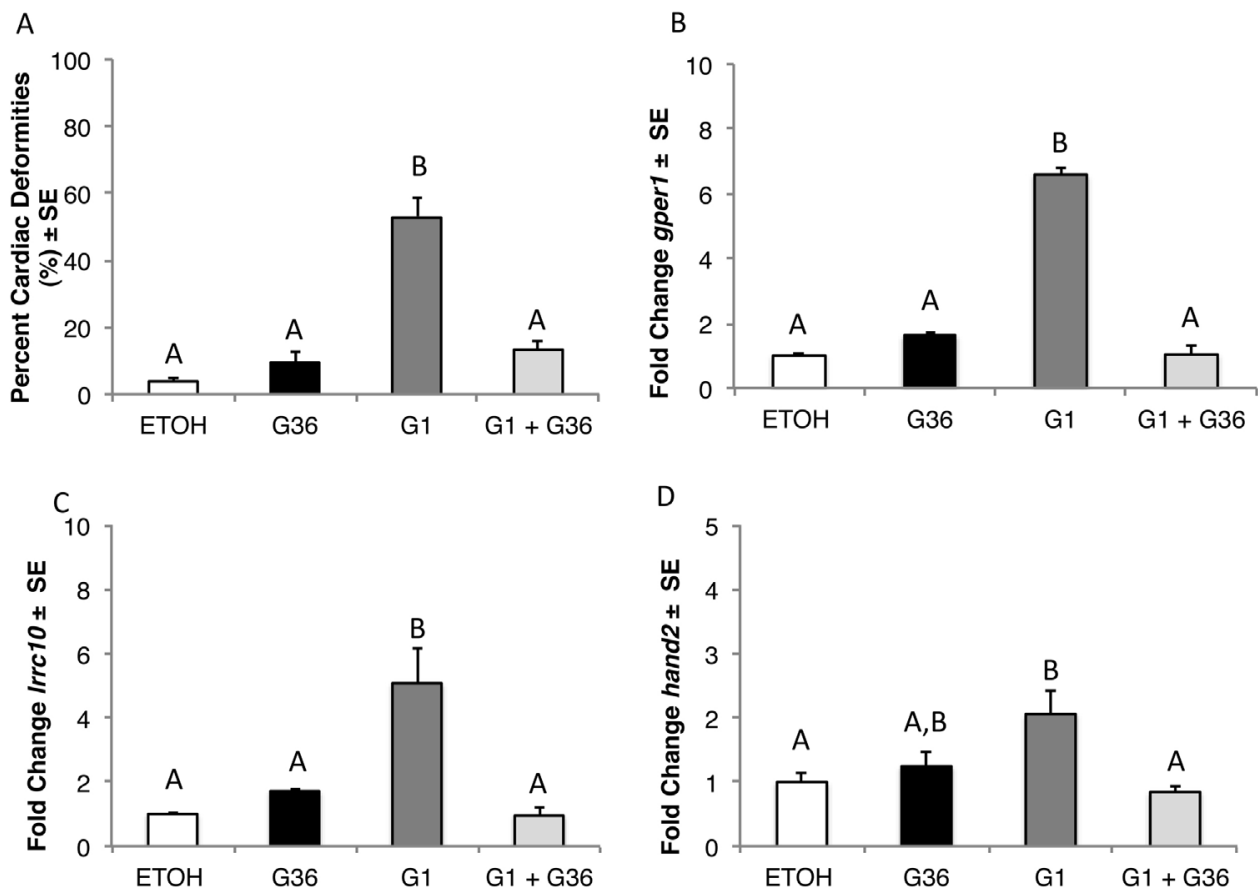

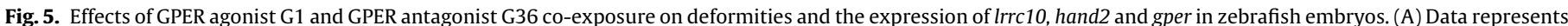

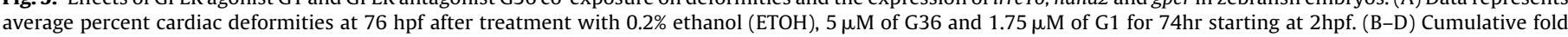

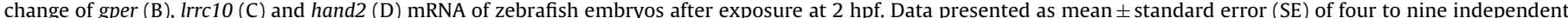

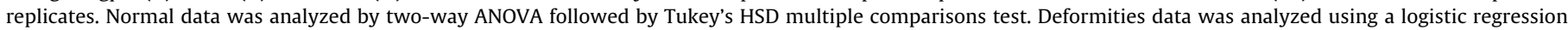
model. Different letters represent the significant difference $(p<0.05)$. 

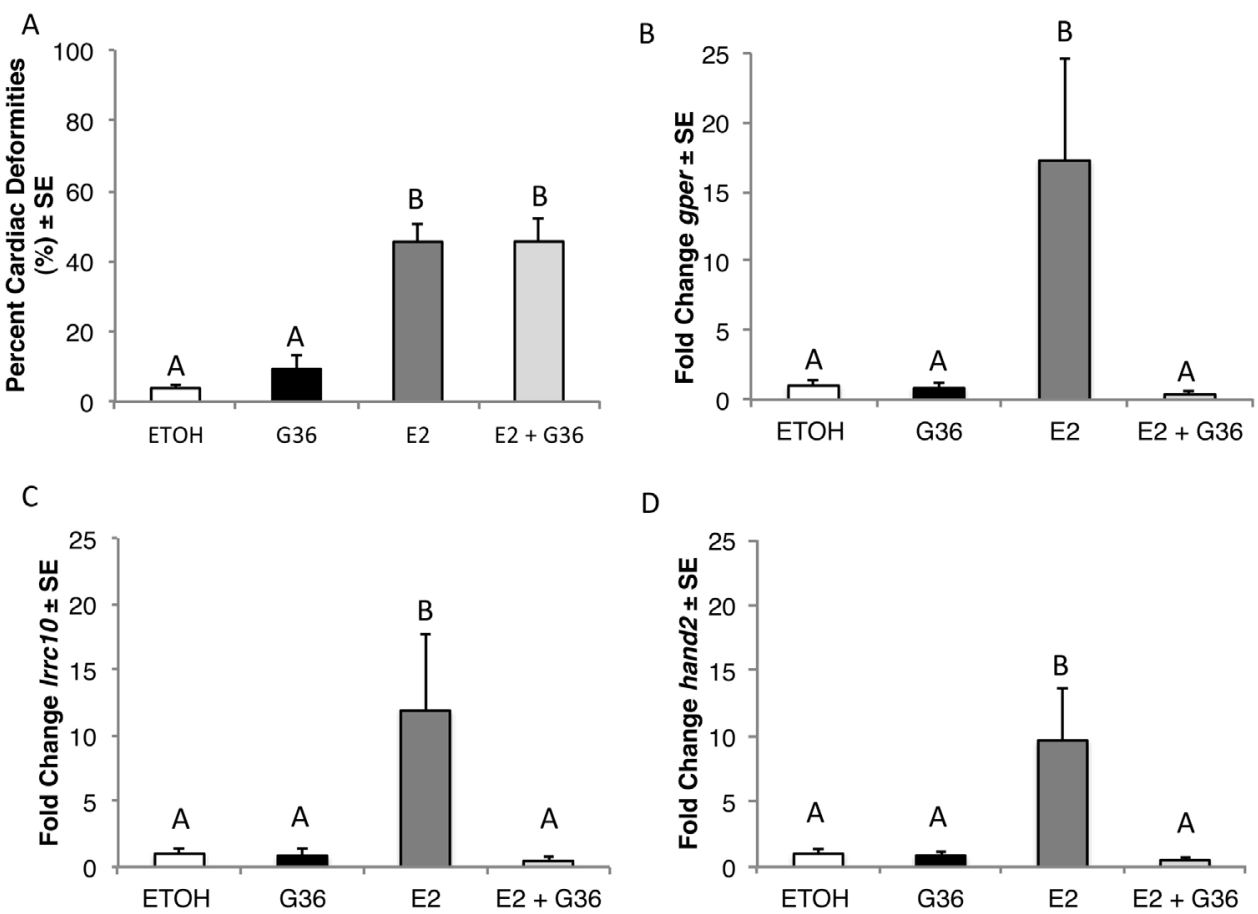

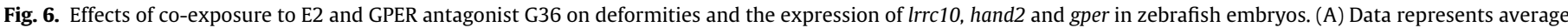

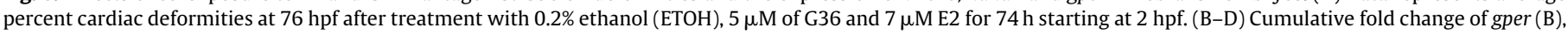

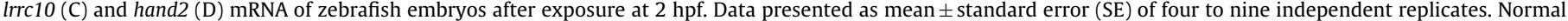

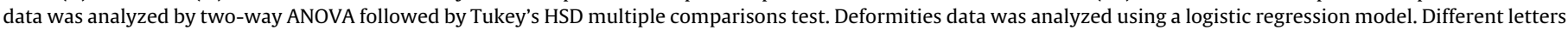
represent the significant difference $(p<0.05)$.

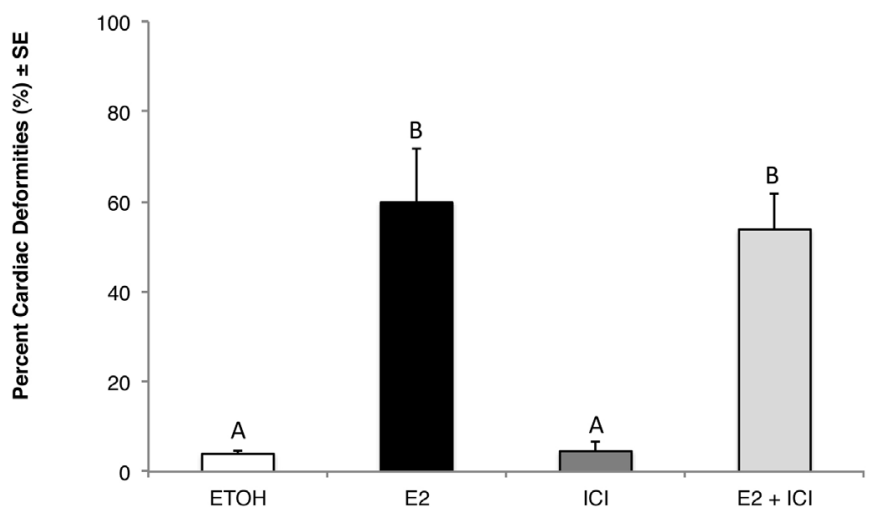

Fig. 7. Percent of cardiac deformities after co-exposure of ER antagonist ICI 182, 780 with $17 \beta$-estradiol (E2). Data represents average percent cardiac deformities at 76 hpf after treatment with $0.2 \%$ ethanol (ETOH), $7 \mu \mathrm{M}$ of E2, $14 \mu \mathrm{M}$ of ICI 182, 780 and $2 \mu \mathrm{M}$ of Ral at $2 \mathrm{hpf}$. Data presented as mean \pm standard error (SE) of four to six independent replicates. Data was analyzed using a logistic regression model. Asterisks $\left({ }^{*}\right)$ represent the significant difference $(p<0.05)$.

development (Irrc10 and hand2). Embryo lethality and developmental abnormalities such as cardiac deformities (e.g., pericardial edema) were temporally related to the altered expression patterns.

A significant increase in cardiac deformities (primarily pericardial edema) was observed after exposure to $7-8 \mu \mathrm{M}$ of E2 at 76 hpf. Exposure to E2 (7-96 hpf) was reported to cause cardiac abnormalities in zebrafish embryos, but was observed at lower concentrations $(2 \mu \mathrm{M})$ (Chandrasekar et al., 2010). The inconsistency in effect thresholds are likely due to experimental design differences such as different treatment vessels, duration and developmental stage. In our study, exposure was only for $74 \mathrm{~h}$ with exposure occurring at 2 hpf. In contrast, Chandrasekar et al. (2010) exposed 7-hpf embryos to $\mathrm{E} 2$ for $96 \mathrm{~h}$. Cardiac deformities have also been observed with other estrogen receptor ligands including EE2, BPA, bifenthrin, and genistein (Duan et al., 2008; Jin et al., 2009; Tse et al., 2013; Santos et al., 2014). EE2 was shown to alter heart rate and cause pericardial edema after exposure for $142 \mathrm{~h}$ (2-144 hpf) (Santos et al., 2014). Exposure to BPA in embryos from 8 to $120 \mathrm{hpf}$ resulted in cardiac abnormalities at concentrations between 30 and $70 \mu \mathrm{M}$ (Saili et al., 2012). Similarly, exposing 24-hpf zebrafish embryos to genistein $(25$ and $50 \mu \mathrm{M})$ for $60 \mathrm{~h}$ resulted in a reduction of heart rate and increased pericardial edema (Kim et al., 2009).

Consistent with earlier studies, G1 also caused similar developmental cardiac toxicity in the current study (Jayasinghe and Volz, 2012). In addition, expression of gper mRNA was significantly increased following E2 treatment. Previous studies have shown that E2 induced the levels of both GPER mRNA and protein in breast cancer cells via the epidermal growth factor receptor (EGFR) pathway (Vivacqua et al., 2009). Overexpression of GPER has been observed in breast and lung cancer cells, indicating its role in different chronic diseases (Filardo et al., 2006; Jala et al., 2012). The role of EGFR in the developmental toxicity of estrogenic compounds in zebrafish is unclear, but warrants further study.

GPER signaling is important for reproductive physiology and cardiovascular function (e.g. vasolidation) (Bopassa et al., 2010; Deschamps and Murphy, 2009; Meyer et al., 2011). In this study, E2 altered hand2 transcripts at $12 \mathrm{hpf}$ and $76 \mathrm{hpf}$ and treatment with $\mathrm{G} 1$ also caused altered expression of hand2. The transcription factor, hand2 belongs to the beta helix-loop-helix (bHLH) family of proteins and regulates cardiac differentiation, especially during early somitogenesis, which occurs $\sim 10-24$ hpf in zebrafish (Kimmel et al., 1995; Stainier, 2001; Yelon et al., 2000). In addition, hand2 plays a role in Notch and NFAT signaling in the endocardium (VanDusen et al., 2014). Overexpression of hand2 in zebrafish embryos increases the number of cardiomyocyte cells and causes heart enlargement (Schindler et al., 2014). For example, mice that overexpressed hand2 developed cardiac hypertrophy (Dirkx et al., 2013). Thus, the increase of hand 2 observed in our study may neg- 
atively affect cardiac development, but further study is needed to test this hypothesis.

Changes in the expression of Irrc10 during development were also observed in the study after exposure to E2 at $12 \mathrm{hpf}$ and 76 hpf. Transcripts of Irrc10 were also altered by G1 exposure, showing that activation of GPER is a potential pathway for E2-induced expression of Irrc10. LRRC10 is a cardiac specific protein that contains leucine-rich repeat (LRR) motifs and is found in mice, humans and zebrafish. LRRC10 also has a significant role in heart function and development in mice as well as zebrafish (Brody et al., 2013; Kim et al., 2007; Nakane et al., 2004). A zebrafish study using lrrc10 morpholinos showed that knocking down this gene caused cardiac morphogenic defects, such as cardiac looping errors, in addition to pericardial edema (Kim et al., 2007). This, along with the results from our study indicates that any disturbance in the levels of lrrc10 may also result in abnormal development.

To verify that the altered gene expression of gper, Irrc10 and hand2 were mediated through the activation of GPER, embryos were co-exposed to the GPER antagonist, G36. Co-exposure of G1 with $\mathrm{G} 36$ rescued the altered expression of all three genes back to control levels. In addition, the rescue of gper, $\operatorname{lrc} 10$ and hand 2 expression was associated with a reduced percentage of cardiac deformities in the G1-G36 co-exposure. This shows that gper, Irrc10 and hand 2 may be involved in the cardiac effects of $\mathrm{G} 1$.

In contrast to G1, G36 was unable to rescue the cardiac deformities induced by E2 exposure, although the expression of gper, hand2 and $\operatorname{lrc} 10$ were reduced in the E2-G36 co-exposure. To investigate the involvement of the estrogen receptor pathway in E2 toxicity, a co-exposure study was conducted using the ER antagonist, ICI 182,780 (ICI). Interestingly, neither ICI 182, 780 nor G36 rescued the observed cardiac effects caused by $\mathrm{E} 2$. While these results were unexpected, it is important to note the limitations of chemical coexposure experiments in evaluating the mechanism of toxicity of compounds. The inability of G36 to rescue E2 cardiac deformities does not eliminate the contribution of other rapid estrogen signaling pathways involvement such as ERs variants (i.e. mER36) that can localize in the membrane (Razandi et al., 1999; Levin, 2002; Chaudhri et al., 2012). In addition, E2 may induce expression of additional ERs that may not be inhibited by ICI. Although these compounds are known bind to specific receptors and initial downstream effects, it is currently not possible to eliminate all other pathways that might be affected by E2 at this concentration, time-point and developmental stage. Thus, the inability of ICI 182,780 or G36 to rescue the E2 induced morphological effects may be due to the possibility that concentrations used for the study were insufficient to reverse E2 effects at the specific stage of development examined. Clearly, concentrations of G36 were sufficient to reduce G1-mediated effects and expression of GPER regulated cardiac genes, but morphological effects of E2 were unchanged. Concentrations of agonists and antagonists for all exposures were optimized based on solubility and overt toxicity. Antagonist concentrations above the solubility limit or that caused overt toxicity were not used. Although other studies in zebrafish adults have shown that $1 \mu \mathrm{M} \mathrm{ICI}$ can rescue effects of $10 \mathrm{nM}$ E2, this was seen in adult male fish (Lam et al., 2011). Given that the concentrations of ICI in our study $(14 \mu \mathrm{M})$ were more than 10 -fold greater that those used in the adult male exposure, and below limits of solubility and overt toxicity, it seems likely that concentrations of ICI should have been high enough to elicit anti-estrogenic activity. In fact, the ICImediated inhibition of $v$ tg induction by E2 is also consistent with ICI concentrations being relevant. However, the kinetic disposition of E2 and antagonists to specific molecular targets in adults relative to embryos is unknown, and suggests morphological effects may be dependent upon the time of exposure.

Alteration in the transcriptional regulation of gper, hand2 and Irrc 10 was observed at $12 \mathrm{hpf}$ and $76 \mathrm{hpf}$ but not at $28 \mathrm{hpf}$. Enhanced expression at unique temporal periods may indicate sensitive windows for E2 toxicity during zebrafish development. At $12 \mathrm{hpf}$, the embryo is in the segmentation period of development. At this stage, cardiac precursor cells begin to move towards the embryonic axis and segregate (Stainier, 2001; Bakkers, 2011). At 28 hpf, ventricular and atrial chambers of the heart tube are becoming more distinct (Stainier, 2001). At $76 \mathrm{hpf}$, rapid growth of all tissues including the heart is occurring (Staudt and Stainier, 2012). This is the time when deformities were observed in the current study. Proliferation of cardiac myocytes, and development of the primitive leaflet valves occur during $76 \mathrm{hpf}$ and are critical for regulating blood flow (Scherz et al., 2008). The degree of reduced blood flow needed to cause embryo or larval mortality is unclear but would be a fruitful area of further study.

Due to the simultaneous increases in Irrc10, hand2 and gper expression after E2 treatment at specific time and developmental stages, the levels of secondary messengers, which theoretically link the downstream genes with GPER were analyzed. $\mathrm{Ca}^{2+}$ and CAMP concentrations did not significantly change after E2 exposure at 76 hpf. These findings suggest that hand2 and lrrc10 expression may not be dependent on significant increases in the concentrations of secondary messengers, suggesting the role of other pathways. It should be noted, however, that the use of whole embryo homogenates also could decrease the signal-to-noise ratio potentially masking site-specific alterations in $\mathrm{Ca}^{2+}$ concentration such as the heart. Many studies have shown that E2 can alter cellular concentrations of $\mathrm{Ca}^{2+}$ but most studies have been conducted in isolated cells, which allow more sensitive detection (Filardo et al., 2002; Revankar et al., 2005; Thomas et al., 2005). Embryonic exposure to $5 \mu \mathrm{M}$ of $\mathrm{G} 1$ resulted in a significant increase of $\mathrm{Ca}^{2+}$ concentrations and a significant decrease in cAMP in embryos even though alterations in downstream gene expression (hand2 and lrrc10) were observed at $2 \mu \mathrm{M}$ and not at $5 \mu \mathrm{M}$ of G1. These findings suggest that activation of these genes via GPER may occur independently or have cell-specific second messenger pathways, which may require microscopic imaging to identify in whole embryos.

In summary, our data suggest that E2-induced cardiotoxicity in developing zebrafish embryos may not be attributed to a specific estrogen pathway. This further raises the question of the role of GPER and ERs during development. Given the widespread occurrence of structurally similar environmental pollutants, such as hydroxy-PAHs or phenolic compounds, that affect estrogen pathways, it is necessary to better characterize this pathway in vertebrate development and environmental toxicology.

\section{Acknowledgments}

This research was made possible by a grant from the Gulf of Mexico Research Initiative, Grant No. SA-1520; Name: Relationship of Effects of Cardiac Outcomes in fish for Validation of Ecological Risk (RECOVER). The authors are grateful to the Gulf of Mexico Research Initiative Information and Data Cooperative (GRIIDC) for supporting data management system to store the data generated. GRIIDC DOI: 10.7266/N7P848X9. The study was also funded through the University of California, Riverside College of Natural and Agricultural Science US Department of Agriculture/Agricultural Experiment Station Resource Support Allocation Program.

\section{Appendix A. Supplementary data}

Supplementary data associated with this article can be found, in the online version, at http://dx.doi.org/10.1016/j.aquatox.2017.02. 024 . 


\section{References}

Bakkers, J., 2011. Zebrafish as a model to study cardiac development and human cardiac disease. Cardiovasc. Res. 91, 279-288.

Bopassa, J.C., Eghbali, M., Toro, L., Stefani, E., 2010. A novel estrogen receptor GPER inhibits mitochondria permeability transition pore opening and protects the heart against ischemia-reperfusion injury. Am. J. Physiol. Heart Circ. Physiol. 298, 16-23.

Brailoiu, G.C., Arterburn, J.B., Oprea, T.I., Chitravanshi, V.C., Brailoiu, E., 2013. Bradycardic effects mediated by activation of $\mathrm{G}$ protein-coupled estrogen receptor in rat nucleus ambiguus. Exp. Physiol. 98, 679-691.

Brion, F., Tyler, C.R., Palazzi, X., Laillet, B., Porcher, J.M., Garric, J., Flammarion, P., 2004. Impacts of 17 beta-estradiol, including environmentally relevant concentrations, on reproduction after exposure during embryo-larval-, juvenile- and adult-life stages in zebrafish (Danio rerio). Aquat. Toxicol. 68, $193-217$.

Brody, M.J., Cho, E., Mysliwiec, M.R., Kim, T.G., Carlson, C.D., Lee, K.H., Lee, Y., 2013. Lrrc10 is a novel cardiac-specific target gene of Nkx2-5 and GATA4. J. Mol. Cell. Cardiol. 62, 37-46.

Chandrasekar, G., Archer, A., Gustafsson, J.A., Andersson Lendahl, M., 2010. Levels of $17 \mathrm{~b}$-estradiol receptors expressed in embryonic and adult zebrafish following in vivo treatment of natural or synthetic ligands. PLoS ONE 5, e9678.

Chaudhri, R.A., Olivares-Navarrete, R., Cuenca, N., Hadadi, A., Boyan, B.D., Schwartz, Z., 2012. Membrane estrogen signaling enhances tumorigenesis and metastatic potential of breast cancer cells via estrogen receptor- $\alpha 36$ (ER $\alpha 36)$. J. Biol. Chem. 287 (March 2 (10)), 7169-7181.

Deschamps, A.M., Murphy, E., 2009. Activation of a novel estrogen receptor, GPER is cardioprotective in male and female rats. Am. J. Physiol. Heart Circ. Physiol. 297, H1806-H1813.

Dirkx, E., Gladka, M.M., Philippen, L.E., Armand, A.S., Kinet, V., Leptidis, S., El Azzouzi, H., Salic, K., Bourajjaj, M., da Silva, G.J., Olieslagers, S., van der Nagel, R., de Weger, R., Bitsch, N., Kisters, N., Seyen, S., Morikawa, Y., Chanoine, C., Heymans, S., Volders, P.G., Thum, T., Dimmeler, S., Cserjesi, P., Eschenhagen, T., da Costa Martins, P.A., De Windt, L.J., 2013. Nfat and miR-25 cooperate to reactivate the transcription factor Hand2 in heart failure. Nat. Cell. Biol. 15, $1282-1293$.

Duan, Z., Zhu, L., Zhu, L., Kun, Y., Zhu, X., 2008. Individual and joint toxic effects of pentachlorophenol and bisphenol A on the development of zebrafish (Danio rerio) embryo. Ecotoxicol. Environ. Saf. 71, 774-780.

Filardo, E.J., Quinn, J.A., Frackelton Jr., A.R., Bland, K.I., 2002. Estrogen action via the G protein-coupled receptor, GPR30: stimulation of adenylyl cyclase and cAMP-mediated attenuation of the epidermal growth factor receptor-to-MAPK signaling axis. Mol. Endocrinol. 16, 70-84

Filardo, E.J., Graeber, C.T., Quinn, J.A., Resnick, M.B., Giri, D., DeLellis, R.A., Steinhoff, M.M., Sabo, E., 2006. Distribution of GPR30, a seven membrane-spanning estrogen receptor, in primary breast cancer and its association with clinicopathologic determinants of tumor progression. Clin. Cancer Res. 12 6359-6366.

Guillette Jr., L.J., Crain, D.A., Rooney, A.A., Pickford, D.B., 1995. Organization versus activation: the role of endocrine-disrupting contaminants (EDCs) during embryonic development in wildlife. Environ. Health Perspect. 103, 157-164

Incardona, J.P., Collier, T.K., Scholz, N.L., 2004. Defects in cardiac function precede morphological abnormalities in fish embryos exposed to polycyclic aromatic hydrocarbons. Toxicol. Appl. Pharmacol. 196, 191-205.

Jala, V.R., Radde, B.N., Haribabu, B., Klinge, C.M., 2012. Enhanced expression of G-protein coupled estrogen receptor (GPER/GPR30) in lung cancer. BMC Cancer 12, 624

Jayasinghe, B.S., Volz, D.C., 2012. Aberrant ligand-induced activation of G protein-coupled estrogen receptor 1 (GPER) results in developmental malformations during vertebrate embryogenesis. Toxicol. Sci. 125, 262-273.

Jin, Y., Chen, R., Sun, L., Qian, H., Liu, W., Fu, Z., 2009. Induction of estrogen-responsive gene transcription in the embryo, larval, juvenile and adult life stages of zebrafish as biomarkers of short-term exposure to endocrine disrupting chemicals. Comp. Biochem. Physiol. C Toxicol. Pharmacol. $150,414-420$.

Kim, D.J., Seok, S.H., Baek, M.W., Lee, H.Y., Na, Y.R., Park, S.H., Lee, H.K., Dutta, N.K., Kawakami, K., Park, J.H., 2009. Developmental toxicity and brain aromatase induction by high genistein concentrations in zebrafish embryos. Toxicol Mech. Methods 19, 251-256.
Kim, K.H., Antkiewicz, D.S., Yan, L., Eliceiri, K.W., Heideman, W., Peterson, R.E., Lee, Y., 2007. Lrrc10 is required for early heart development and function in zebrafish. Dev. Biol. 308, 494-506.

Kimmel, C.B., Ballard, W.W., Kimmel, S.R., Ullmann, B., Schilling, T.F., 1995. Stages of embryonic development of the zebrafish. Dev. Dyn. 203, 253-310.

Lam, S.H., Lee, S.G., Lin, C.Y., Thomsen, J.S., Fu, P.Y., Murthy, K.R., Li, H., Govindarajan, K.R., Nick, L.C., Bourque, G., Gong, Z., Lufkin, T., Liu, E.T., Mathavan, S., 2011. Molecular conservation of estrogen-response associated with cell cycle regulation, hormonal carcinogenesis and cancer in zebrafish and human cancer cell lines. BMC Med. Genomics 4, 41.

Levin, E.R., 2002. Cellular functions of plasma membrane estrogen receptors. Steroids 67 (6), 471-475.

Meyer, M.R., Prossnitz, E.R., Barton, M., 2011. The G protein-coupled estrogen receptor GPER/GPR30 as a regulator of cardiovascular function. Vascul. Pharmacol. 55, 17-25.

Nakane, T., Satoh, T., Inada, Y., Nakayama, J., Itoh, F., Chiba, S., 2004. Molecular cloning and expression of HRLRRP, a novel heart-restricted leucine-rich repeat protein. Biochem. Biophys. Res. Commun. 314, 1086-1092.

Razandi, M., Pedram, A., Greene, G.L., Levin, E.R., 1999. Cell membrane and nuclear estrogen receptors (ERs) originate from a single transcript: studies of ERalpha and ERbeta expressed in Chinese hamster ovary cells. Mol Endocrinol. 13 (2), 307-319.

Revankar, C.M., Cimino, D.F., Sklar, L.A., Arterburn, J.B., Prossnitz, E.R., 2005. A transmembrane intracellular estrogen receptor mediates rapid cell signaling. Science 307, 1625-1630.

Saili, K.S., Corvi, M.M., Weber, D.N., Patel, A.U., Das, S.R., Przybyla, J., Anderson, K.A., Tanguay, R.L., 2012. Neurodevelopmental low-dose bisphenol A exposure leads to early life-stage hyperactivity and learning deficits in adult zebrafish. Toxicology 291, 83-92.

Santos, D., Matos, M., Coimbra, A.M., 2014. Developmental toxicity of endocrine disruptors in early life stages of zebrafish, a genetic and embryogenesis study. Neurotoxicol. Teratol. 46, 18-25.

Scherz, P.J., Huisken, J., Sahai-Hernandez, P., Stainier, D.Y., 2008. High-speed imaging of developing heart valves reveals interplay of morphogenesis and function. Development 135, 1179-1187.

Schindler, Y.L., Garske, K.M., Wang, J., Firulli, B.A., Firulli, A.B., Poss, K.D., Yelon, D. 2014. Hand2 elevates cardiomyocyte production during zebrafish heart development and regeneration. Development 141, 3112-3122.

Sonnenschein, C., Soto, A.M., 1998. An updated review of environmental estrogen and androgen mimics and antagonists. J. Steroid Biochem. Mol. Biol. 65 $143-150$.

Stainier, D.Y., 2001. Zebrafish genetics and vertebrate heart formation. Nat. Rev. Genet. 2, 39-48

Staudt, D., Stainier, D., 2012. Uncovering the molecular and cellular mechanisms of heart development using the zebrafish. Annu. Rev. Genet. 46, 397-418.

Szego, C.M., Davis, J.S., 1967. Adenosine 3',5'-monophosphate in rat uterus: acute elevation by estrogen. Proc. Natl. Acad. Sci. U.S.A. 58, 1711-1718.

Thomas, P., Pang, Y., Filardo, E.J., Dong, J., 2005. Identity of an estrogen membrane receptor coupled to a $\mathrm{G}$ protein in human breast cancer cells. Endocrinology 146, 624-632.

Tse, W.K., Yeung, B.H., Wan, H.T., Wong, C.K., 2013. Early embryogenesis in zebrafish is affected by bisphenol A exposure. Biol. Open. 2, 466-471

VanDusen, N.J., Casanovas, J., Vincentz, J.W., Firulli, B.A., Osterwalder, M., Lopez-Rios, J., Zeller, R., Zhou, B., Grego-Bessa, J., De La Pompa, J.L., Shou, W., Firulli, A.B., 2014. Hand2 is an essential regulator for two Notch-dependent functions within the embryonic endocardium. Cell Rep. 9, 2071-2083.

Vivacqua, A., Lappano, R., De Marco, P., Sisci, D., Aquila, S., De Amicis, F., Fuqua, S.A., Andò, S., Maggiolini, M., 2009. G protein-coupled receptor 30 expression is up-regulated by EGF and TGF alpha in estrogen receptor alpha-positive cancer cells. Mol. Endocrinol. 23, 1815-1826.

Yelon, D., Ticho, B., Halpern, M.E., Ruvinsky, I., Ho, R.K., Silver, L.M., Stainier, D.Y., 2000. The bHLH transcription factor hand2 plays parallel roles in zebrafish heart and pectoral fin development. Development 127, 2573-2582.

Zhao, S., Fernald, R.D., 2005. Comprehensive algorithm for quantitative real-time polymerase chain reaction. J. Comput. Biol. 12 (8), 1047-1064. 